\title{
Monogenic Functions in Conformal Geometry ${ }^{\star}$
}

\author{
Michael EASTWOOD ${ }^{\dagger}$ and John RYAN ${ }^{\ddagger}$ \\ $\dagger$ Department of Mathematics, University of Adelaide, SA 5005, Australia \\ E-mail: meastwoo@member.ams.org \\ $¥$ Department of Mathematics, University of Arkansas, Fayetteville, AR 72701, USA \\ E-mail: jryan@uark.edu
}

Received August 29, 2007; Published online August 30, 2007

Original article is available at http://www.emis.de/journals/SIGMA/2007/084/

\begin{abstract}
Monogenic functions are basic to Clifford analysis. On Euclidean space they are defined as smooth functions with values in the corresponding Clifford algebra satisfying a certain system of first order differential equations, usually referred to as the Dirac equation. There are two equally natural extensions of these equations to a Riemannian spin manifold only one of which is conformally invariant. We present a straightforward exposition.
\end{abstract}

Key words: Clifford analysis; monogenic functions; Dirac operator; conformal invariance 2000 Mathematics Subject Classification: 53A30; 58J70; 15A66

\section{Introduction}

This article is dedicated to the memory of Tom Branson, a true gentleman, scholar, and friend. A significant and recurring theme in his work was the interaction between the following topics

\begin{tabular}{|c|c|c|c|c|}
\hline $\begin{array}{l}\text { Lie Groups } \\
\text { Global Symmetry }\end{array}$ & $\begin{array}{l}\text { Homogeneous Spaces } \\
\text { Representation Theory }\end{array}$ & $\leftarrow$ & $\begin{array}{l}\text { Differential Geometry } \\
\text { Local Symmetry }\end{array}$ & $\begin{array}{l}\text { Local Structure } \\
\text { PDEs }\end{array}$ \\
\hline
\end{tabular}

Items in the left hand box are concerned with geometry and global analysis on a homogeneous space $G / P$ where $G$ is a Lie group with subgroup $P$. Items in the right hand box are concerned with local differential geometry 'modelled' on the homogeneous space $G / P$. Without trying to explain what this might mean in general, one of the best known examples is to have conformal differential geometry in dimension $n \geq 3$ in the right hand box and the sphere $S^{n}$, viewed as a homogeneous space for $\mathrm{SO}(n+1,1)$, in the left hand box. The round sphere provides a better 'flat model' of conformal geometry than does Euclidean space because all the conformal Killing fields integrate to genuine conformal motions (and there is a conformal embedding $\mathbb{R}^{n} \hookrightarrow S^{n}$ given by inverse stereographic projection). Tom Branson was one of the first (e.g. [4]) systematically to exploit the interplay between 'curved' conformal differential geometry and its flat model. He was also keen to promote the interaction between conformal geometry and the field of Clifford analysis, where monogenic functions were defined and are now studied.

Some disclaimers are in order. The results in this article are, in some sense, already known. More precisely, there are a few mathematicians, including David Calderbank and Vladimír Souček, who are well aware of results in this vein. There is no doubt that Tom Branson would have had his own distinctive viewpoint. Our aim, therefore, is to give what we believe to be a particularly efficient formulation and method of proof with minimal prerequisites. Motivation and consequences will be given as we go along.

\footnotetext{
${ }^{\star}$ This paper is a contribution to the Proceedings of the 2007 Midwest Geometry Conference in honor of Thomas P. Branson. The full collection is available at http://www.emis.de/journals/SIGMA/MGC2007.html
} 
Clifford analysis on Euclidean space $\mathbb{R}^{n}$ is usually developed by considering smooth functions with values in the Clifford algebra of $\mathbb{R}^{n}$. In this context one may equally well consider smooth differential forms on $\mathbb{R}^{n}$, i.e. smooth functions with values in $\Lambda^{\bullet}=\bigoplus_{p=0}^{n} \Lambda^{p}$ where $\Lambda^{p}$ is the bundle of $p$-forms. In fact, there is a canonical identification between $\Lambda^{\bullet} \mathbb{R}^{n}$ and the Clifford algebra. Under this identification, the so-called 'Dirac operator' $D: \Lambda^{\bullet} \rightarrow \Lambda^{\bullet}$ is precisely

$$
D \omega=d \omega-d^{*} \omega
$$

where $d$ is exterior derivative and $d^{*}$ is its adjoint. Viewed like this, $D$ enjoys an evident extension to any Riemannian manifold where it is called the Hodge-de Rham operator. There is, however, an equally natural but different extension of $D$ to any Riemannian manifold with spin structure. This alternative extension turns out to be conformally invariant and hence to be preferred as the starting point for Clifford analysis on manifolds.

In this article, we shall present this alternative extension in as simple a manner as possible. In particular, we shall not need Clifford algebras for the basic exposition, only to compare the resulting Dirac operator with the classical one defined on the usual spin bundles in $\S 4$ (and, even then, only implicitly). The simplest exposition does not even require any representation theory beyond the basics.

We conclude this introduction by establishing some notation and conventions. We shall use Penrose's 'abstract index notation' [17] for tensors on a manifold and also for representations of the orthogonal group $\mathrm{SO}(n)$. Thus, we shall write $\omega_{a}$ to denote a smooth 1 -form, and $X^{a}$ to denote a vector field. Square brackets denote skewing over the indices they enclose - if $\omega_{a b}=\omega_{[a b]}$, then $\omega_{a b}$ denotes a 2-form. Similarly, we shall use round brackets to denote symmetrisation: $\phi_{(a b)}=\frac{1}{2}\left(\phi_{a b}+\phi_{b a}\right)$. Repeated indices denote the natural pairing of vectors and co-vectors: $X\lrcorner \omega=X^{a} \omega_{a}$. In local coördinates this is the Einstein summation convention but the abstract index convention is itself coördinate free. Following standard practise, on a Riemannian manifold we shall write $g_{a b}$ for the metric and $g^{a b}$ for its inverse: $g_{a b} g^{b c}=\delta_{a}{ }^{c}$ where $\delta_{a}{ }^{c}$ is the Kronecker delta or identity matrix. We shall often 'raise and lower indices' without comment if $X^{a}$ is a vector field then $X_{a} \equiv g_{a b} X^{b}$ is the corresponding 1-form. Sometimes we shall write $\langle u, v\rangle$ instead of $u^{a} v_{a}$. We need to set some conventions for the exterior and interior product on differential forms - if $\omega_{b c \cdots d}$ has $p$ indices, then

$$
\left.(v \wedge \omega)_{a b c \cdots d} \equiv v_{[a} \omega_{b c \cdots d]} \quad \text { and } \quad(v\lrcorner \omega\right)_{c \cdots d} \equiv p v^{b} \omega_{b c \cdots d} .
$$

These conventions have the convenient feature that

$$
u \wedge(v\lrcorner \omega)+v\lrcorner(u \wedge \omega)=\langle u, v\rangle \omega .
$$

For further details see [18].

\section{Conformally invariant first order operators}

We begin with three simple examples. If $\omega_{a}$ is a 1-form on a Riemannian manifold $M$ and we rescale the metric according to $\hat{g}_{a b}=\Omega^{2} g_{a b}$, then it is easily verified that

$$
\hat{\nabla}_{a} \omega_{b}=\nabla_{a} \omega_{b}-\Upsilon_{a} \omega_{b}-\Upsilon_{b} \omega_{a}+\Upsilon^{c} \omega_{c} g_{a b} \quad \text { where } \Upsilon_{a}=\nabla_{a} \Omega / \Omega
$$

and $\nabla_{a}$ is the metric connection for $g_{a b}$ whilst $\hat{\nabla}_{a}$ is the metric connection for $\hat{g}_{a b}$. It follows that

$$
\hat{\nabla}_{[a} \omega_{b]}=\nabla_{[a} \omega_{b]}
$$


This is just the conformal invariance of the exterior derivative $d: \Lambda^{1} \rightarrow \Lambda^{2}$. More instructive examples are obtained by insisting that when we rescale the metric so that $\hat{g}_{a b}=\Omega^{2} g_{a b}$ we also rescale the 1 -form $\hat{\omega}_{a}=\Omega^{w} \omega_{a}$. In this case

$$
\begin{aligned}
\hat{\nabla}_{a} \hat{\omega}_{b} & =\hat{\nabla}_{a}\left(\Omega^{w} \omega_{b}\right) \\
& =\left(\nabla_{a} \Omega^{w}\right) \omega_{b}+\Omega^{w} \hat{\nabla}_{a} \omega_{b} \\
& =\Omega^{w} w \Upsilon_{a} \omega_{b}+\Omega^{w}\left(\nabla_{a} \omega_{b}-\Upsilon_{a} \omega_{b}-\Upsilon_{b} \omega_{a}+\Upsilon^{c} \omega_{c} g_{a b}\right) \\
& =\Omega^{w}\left(\nabla_{a} \omega_{b}+(w-1) \Upsilon_{a} \omega_{b}-\Upsilon_{b} \omega_{a}+\Upsilon^{c} \omega_{c} g_{a b}\right) .
\end{aligned}
$$

Notice that the right hand side rescales by the same power of $\Omega$ as we decreed for our 1-form $\omega_{a}$. Thus, if we say that $\omega_{a}$ is conformally weighted of weight $w$ instead of writing $\hat{\omega}_{a}=\Omega^{w} \omega_{a}$, then we have shown that

$$
\hat{\nabla}_{a} \omega_{b}=\nabla_{a} \omega_{b}+(w-1) \Upsilon_{a} \omega_{b}-\Upsilon_{b} \omega_{a}+\Upsilon^{c} \omega_{c} g_{a b}
$$

for $\omega_{a}$ of conformal weight $w$. More precisely, we define a conformal manifold to be a smooth manifold equipped with an equivalence class of Riemannian metrics under the equivalence relation $g_{a b} \mapsto \hat{g}_{a b}=\Omega^{2} g_{a b}$ for any smooth function $\Omega$. On such a manifold we define a line bundle $L$ as trivialised by any choice of metric - its sections are identified as ordinary functions $f$. For a conformally related metric $\hat{g}_{a b}=\Omega^{2} g_{a b}$, however, the same section gives a different function $\hat{f}=\Omega f$. Let us write $\Lambda^{0}[w]$ instead of $L^{w}$ and refer to its sections as conformal densities of weight $w$. Similarly, $\Lambda^{p}[w]=\Lambda^{p} \otimes L^{w}$ is the bundle of conformally weighted $p$-forms of weight $w$. For any metric $g_{a b}$ in the conformal class these bundles acquire natural connections, namely by trivialising $L$ and employing the metric connection. Equation (3) now has a precise interpretation - it says how the connection $\nabla: \Lambda^{1}[w] \rightarrow \Lambda^{1} \otimes \Lambda^{1}[w]$ changes under a conformal rescaling of the metric. The metric $g_{a b}$ itself acquires a tautological interpretation as a conformally invariant section of $\bigodot^{2} \Lambda^{1}[2]$. Similarly, its inverse $g^{a b}$ has conformal weight -2 and we may raise and lower indices at the expense of changing the weight - if $\omega_{a}$ has weight $w$, then $\omega^{a}$ has weight $w-2$.

If $w=2$, then (3) reads

$$
\hat{\nabla}_{a} \omega_{b}=\nabla_{a} \omega_{b}+\Upsilon_{a} \omega_{b}-\Upsilon_{b} \omega_{a}+\Upsilon^{c} \omega_{c} g_{a b}
$$

It follows that

$$
\hat{\nabla}_{(a} \omega_{b)}-\frac{1}{n} \hat{\nabla}^{c} \omega_{c} g_{a b}=\nabla_{(a} \omega_{b)}-\frac{1}{n} \nabla^{c} \Upsilon_{c} g_{a b}
$$

Thus, we have a conformally invariant operator

$$
\Lambda^{1}[2] \rightarrow \bigodot_{\circ}^{2} \Lambda^{1}[2]
$$

where $\bigodot_{\circ}^{2} \Lambda^{1}$ denote the trace-free symmetric covariant 2 -tensors.

Taking the trace of (3) yields

$$
\hat{\nabla}^{a} \omega_{a}=\nabla^{a} \omega_{a}+(n+w-2) \Upsilon^{a} \omega_{a} .
$$

We obtain, as our third example, a conformally invariant differential operator

$$
\Lambda^{1}[-(n-2)] \rightarrow \Lambda^{0}[-n]
$$

Our fundamental theorem constructs first order conformally invariant operators from representations of $\mathrm{SO}(n)$ or $\operatorname{Spin}(n)$. Let us denote by $\mathbb{W}$, the defining representation of $\mathrm{SO}(n)$ on $\mathbb{R}^{n}$ and by $g_{a b} \in \mathbb{W} \otimes \mathbb{W}$ or by $\langle$,$\rangle the standard Euclidean metric, preserved by \mathrm{SO}(n)$. We shall 
identify $\Lambda^{2} \mathbb{W}$ with the Lie algebra $\mathfrak{s o}(n)$ of $\operatorname{SO}(n)$ or $\operatorname{Spin}(n)$. Explicitly, let us take the action of $X_{a b} \in \Lambda^{2} \mathbb{W}$ on $w_{a} \in \mathbb{W}$ to be given by $X_{a}^{b} w_{b}$. Also let

$$
w_{a} \mapsto-2 g_{a[b} w_{c]} \quad \text { define } \quad \iota: \mathbb{W} \rightarrow \mathbb{W} \otimes \Lambda^{2} \mathbb{W} .
$$

A representation $\mathbb{E}$ of $\mathrm{SO}(n)$ gives rise to an irreducible tensor bundle $E$ on any oriented Riemannian $n$-manifold. Specifically, we shall regard $E$ as induced from the orthonormal co-frame bundle so that $\mathbb{W}$ gives rise to $\Lambda^{1}$, the co-tangent bundle. Similarly, if $\mathbb{E}$ is a $\operatorname{Spin}(n)$-representation, then we find induced bundles $E$ on any oriented Riemannian spin $n$-manifold. On a conformal manifold it is convenient, following Calderbank [7], to induce bundles from the orthonormal frames of $\Lambda^{1}[1]$. The reason is that $\langle\omega, \mu\rangle=g^{a b} \omega_{a} \mu_{b}$ is conformally invariant for $\omega_{a}$ and $\mu_{a}$ of conformal weight 1 and so these frames are well-defined. A further reason is that, although there is no conformally invariant connection on $\Lambda^{1}[1]$, the conformal change (3) is especially simple:

$$
\hat{\nabla}_{a} \omega_{b}=\nabla_{a} \omega_{b}-\Upsilon_{b} \omega_{a}+\Upsilon^{c} \omega_{c} g_{a b}=\nabla_{a} \omega_{b}-\Gamma_{a b}{ }^{c} \omega_{c} \quad \text { where } \quad \Gamma_{a b c}=-2 g_{a[b} \Upsilon_{c]} .
$$

We shall denote by $E$ the bundle induced on an oriented conformal manifold from a representation $\mathbb{E}$ of $\mathrm{SO}(n)$. From the defining representation $\mathbb{W}$ of $\mathrm{SO}(n)$ we obtain the bundle $\Lambda^{1}[1]$.

Theorem 1. Suppose that $\mathbb{E}$ and $\mathbb{F}$ are representations of $\mathrm{SO}(n)$ and that

$$
\pi: \mathbb{W} \otimes \mathbb{E} \rightarrow \mathbb{F}
$$

is a homomorphism of $\mathrm{SO}(n)$-modules. Let $\rho: \Lambda^{2} \mathbb{W} \otimes \mathbb{E} \rightarrow \mathbb{E}$ denote the action of $\mathfrak{s o}(n)$ on $\mathbb{E}$. Suppose that the composition

$$
\mathbb{W} \otimes \mathbb{E} \stackrel{\iota \otimes \mathrm{Id}}{\longrightarrow} \mathbb{W} \otimes \Lambda^{2} \mathbb{W} \otimes \mathbb{E} \stackrel{\mathrm{Id} \otimes \rho}{\longrightarrow} \mathbb{W} \otimes \mathbb{E} \stackrel{\pi}{\rightarrow} \mathbb{F}
$$

is equal to $w \pi: \mathbb{W} \otimes \mathbb{E} \rightarrow \mathbb{F}$ for some constant $w$. Then there is a conformally invariant first order linear differential operator

$$
D: E[w] \rightarrow F[w-1]
$$

whose symbol is induced by $\pi$. If, instead, $\mathbb{E}$ and $\mathbb{F}$ are representations of $\operatorname{Spin}(n)$, then we obtain the corresponding conclusion on any Riemannian spin manifold.

Proof. Recall the formula (4) for a change of connection on $\Lambda^{1}[1]$. Therefore, for sections $\phi$ of the associated bundle $E$,

$$
\hat{\nabla} \phi=\nabla \phi-\Gamma \phi
$$

where $\Gamma \phi$ is the image of $\Upsilon \otimes \phi$ under the composition

$$
\Lambda^{1} \otimes E \stackrel{\iota \otimes \mathrm{Id}}{\longrightarrow} \Lambda^{1} \otimes \Lambda^{2} \otimes E \stackrel{\mathrm{Id} \otimes \rho}{\longrightarrow} \Lambda^{1} \otimes E .
$$

For sections of $E[w]$, therefore,

$$
\hat{\nabla} \phi=\nabla \phi+w \Upsilon \otimes \phi-\Gamma \phi .
$$

Hence, for $D=\pi \nabla$ we have

$$
\hat{D} \phi=D \phi+w \pi(\Upsilon \otimes \phi)-\pi \Gamma \phi=D \phi,
$$

as required. 
The three conformally invariant operators given at the beginning of this section are easily obtained from Theorem 1. Specifically, the irreducible decomposition

$$
\mathbb{W} \otimes \mathbb{W}=\Lambda^{2} \mathbb{W} \oplus \bigodot_{\circ}^{2} \mathbb{W} \oplus \mathbb{R}
$$

is given explicitly by

$$
\xi_{a} \omega_{b}=\xi_{[a} \omega_{b]}+\left(\xi_{(a} \omega_{b)}-\frac{1}{n} \xi^{c} \omega_{c} g_{a b}\right)+\frac{1}{n} \xi^{c} \omega_{c} g_{a b}
$$

from which we obtain three possible symbols with $\mathbb{E}=\mathbb{W}$ so that $E=\Lambda^{1}[1]$. The composition

$$
\mathbb{W} \otimes \mathbb{E} \stackrel{\iota \otimes \mathrm{Id}}{\longrightarrow} \mathbb{W} \otimes \Lambda^{2} \mathbb{W} \otimes \mathbb{E} \stackrel{\mathrm{Id} \otimes \rho}{\longrightarrow} \mathbb{W} \otimes \mathbb{E}
$$

is $\xi_{a} \omega_{b} \mapsto-2 g_{a[b} \xi_{c]} \omega_{d} \mapsto \xi_{b} \omega_{a}-g_{a b} \xi^{c} \omega_{c} \equiv \sigma_{a b}$ and to apply Theorem 1 we must see whether $\pi \sigma$ is a multiple of $\pi(\xi \otimes \omega)$. We find

$$
\begin{array}{lll}
\xi_{a} \omega_{b} \stackrel{\pi}{\longmapsto} \xi_{[a} \omega_{b]} & \text { gives } \quad \sigma_{[a b]}=-\xi_{[a} \omega_{b]}, \\
\xi_{a} \omega_{b} \stackrel{\pi}{\longmapsto} \xi_{(a} \omega_{b)}-\frac{1}{n} \xi^{c} \omega_{c} g_{a b} & \text { gives } \quad \sigma_{(a b)}-\frac{1}{n} \sigma^{c}{ }_{c} g_{a b}=\left(\xi_{(a} \omega_{b)}-\frac{1}{n} \xi^{c} \omega_{c} g_{a b}\right), \\
\xi_{a} \omega_{b} \stackrel{\pi}{\longmapsto} \xi^{a} \omega_{a} & \text { gives } \quad \sigma_{a}^{a}=-(n-1) \xi^{a} \omega_{a} .
\end{array}
$$

We obtain our three basic conformally invariant operators

\begin{tabular}{c|c|c|c|c}
$\mathbb{F}$ & $w$ & $F$ & $E[w]$ & $F[w-1]$ \\
\hline \hline$\Lambda^{2} \mathbb{W}$ & -1 & $\Lambda^{2}[2]$ & $\Lambda^{1}$ & $\Lambda^{2}$ \\
\hline$\bigodot_{\circ}^{2} \mathbb{W}$ & 1 & $\bigodot_{\circ}^{2} \Lambda^{1}[2]$ & $\Lambda^{1}[2]$ & $\bigodot_{\circ}^{2} \Lambda^{1}[2]$ \\
\hline $\mathbb{R}$ & $-(n-1)$ & $\Lambda^{0}$ & $\Lambda^{1}[-(n-2)]$ & $\Lambda^{0}[-n]$
\end{tabular}

in accordance with Theorem 1.

In stating Theorem 1 we were imprecise concerning whether $\mathbb{E}$ and $\mathbb{F}$ should be real or complex representations of $\mathrm{SO}(n)$ or $\operatorname{Spin}(n)$. In fact, there are two versions - if the representations are real (as in our three examples), then the corresponding induced bundles are real but if the representations are complex (as is more usual in representation theory) then the bundles are complex.

The hypotheses of Theorem 1 are automatically satisfied if $\mathbb{E}$ is an irreducible complex representation of $\mathrm{SO}(n)$ or $\operatorname{Spin}(n)$. In this case the decomposition of $\mathbb{W} \otimes \mathbb{E}$ into irreducibles is multiplicity-free. Thus, if $\pi: \mathbb{W} \otimes \mathbb{E} \rightarrow \mathbb{F}$ is projection onto any of the irreducibles, then any other $\mathfrak{s o}(n)$-invariant homomorphism $\mathbb{W} \otimes \mathbb{E} \rightarrow \mathbb{E}$ must be a multiple thereof. In this case, it is just a matter of identifying the constant $w$ in order to classify the first order conformally invariant differential operators between irreducible tensor or spinor bundles. This is precisely what Fegan does in [10], using Casimir operators to compute w. In [8], Čap, Slovák, and Souček extended Fegan's method to higher order operators.

\section{Monogenic functions and Dirac operators}

The construction and conformal invariance of monogenic functions also comes from Theorem 1. In distinction to the discussion at the end of the previous section, the representation $\mathbb{E}$ will be real and reducible. As a vector space, let $\mathbb{E}=\Lambda^{\bullet} \mathbb{W}$ where, as usual, $\mathbb{W}$ is the (real) defining representation of $\mathrm{SO}(n)$. Define a homomorphism

$$
\mathbb{W} \otimes \mathbb{E} \stackrel{\epsilon}{\rightarrow} \mathbb{E} \quad \text { by } \quad v \otimes e \mapsto v . e \equiv v \wedge e-v\lrcorner e .
$$


There are two natural ways in which $\mathbb{E}$ is an $\mathfrak{s o}(n)$-module. The more obvious corresponds to the $\mathrm{SO}(n)$-action defined by that on $\mathbb{W}$. Evidently, $\epsilon$ is a homomorphism of $\mathrm{SO}(n)$-modules. The geometric consequence of this is a differential operator from $E$ to itself defined on any Riemannian manifold as the composition

$$
E \stackrel{\nabla}{\longrightarrow} \Lambda^{1} \otimes E \stackrel{\epsilon}{\rightarrow} E
$$

where $\nabla$ is induced by the metric connection on the co-frame bundle. This is the Hodge-de Rham operator. For this choice of $\rho: \Lambda^{2} \mathbb{W} \otimes \mathbb{E} \rightarrow \mathbb{E}$, however,

$$
\mathbb{W} \otimes \mathbb{E} \stackrel{\iota \otimes \mathrm{Id}}{\longrightarrow} \mathbb{W} \otimes \Lambda^{2} \mathbb{W} \otimes \mathbb{E} \stackrel{\operatorname{Id} \otimes \rho}{\longrightarrow} \mathbb{W} \otimes \mathbb{E} \stackrel{\epsilon}{\rightarrow} \mathbb{E}
$$

is not a multiple of $\epsilon: \mathbb{W} \otimes \mathbb{E} \rightarrow \mathbb{E}$. The Hodge-de Rham operator is not conformally invariant.

An alternative but equally natural way in which $\mathbb{E}$ is an $\mathfrak{s o}(n)$-module is given by

$$
\mathfrak{s o}(n) \otimes \mathbb{E}=\Lambda^{2} \mathbb{W} \otimes \mathbb{E} \ni v \wedge w \otimes e \stackrel{\sigma}{\longmapsto}-\frac{1}{8}(v . w . e-w . v . e) \in \mathbb{E} .
$$

The constant $-1 / 8$ ensures that this is, indeed, a representation of $\mathfrak{s o}(n)$. To verify this and other properties of this construction, the following lemmata are useful.

Lemma 1. For all $u, v \in \mathbb{W}$ and $e \in \mathbb{E}$ we have

$$
\text { u.v.e +v.u.e }=-2\langle u, v\rangle e .
$$

Proof. A simple computation from (2).

Lemma 2. For all $u, v, w \in \mathbb{W}$ and $e \in \mathbb{E}$ we have

$$
\text { u.v.w.e - v.w.u.e - u.w.v.e +w.v.u.e }=-4\langle u, v\rangle w . e+4\langle u, w\rangle v . e .
$$

Proof. From (7) we find

$$
\begin{aligned}
u . v . w . e-v . w . u . e & =u . v \cdot w \cdot e+v \cdot u \cdot w \cdot e-v \cdot u \cdot w \cdot e-v \cdot w \cdot u \cdot e \\
& =-2\langle u, v\rangle w . e+2\langle u, w\rangle v . e .
\end{aligned}
$$

The other terms in (8) are dealt with similarly.

Proposition 1. The action (6) makes $\mathbb{E}$ into a representation of $\mathfrak{s o}(n)$. With this structure $\epsilon: \mathbb{W} \otimes \mathbb{E} \rightarrow \mathbb{E}$ is a homomorphism of $\mathfrak{s o}(n)$-modules.

Proof. On simple vectors, the Lie bracket on $\mathfrak{s o}(n)=\Lambda^{2} \mathbb{W}$ is given by

$$
[t \wedge u, v \wedge w]=\frac{1}{2}(\langle u, v\rangle t \wedge w-\langle u, w\rangle t \wedge v-\langle t, v\rangle u \wedge w+\langle t, w\rangle u \wedge v) .
$$

The assertions are straightforward calculations on simple vectors using (8).

Lemma 3. If we define $\mathbb{W} \otimes \mathbb{W} \otimes \mathbb{E} \rightarrow \mathbb{E}$ by $v \otimes w \otimes e \mapsto v . w . e$, then $g_{a b} \otimes e \mapsto-n e$.

Proof. If $u_{1}, u_{2}, \ldots, u_{n}$ is an orthonormal basis of $\mathbb{W}$, then $g_{a b}=\sum_{i, j=1}^{n} u_{i} \otimes u_{j}$. The result follows immediately from (7).

Proposition 2. The composition

$$
\mathbb{W} \otimes \mathbb{E} \stackrel{\iota \otimes \mathrm{Id}}{\longrightarrow} \mathbb{W} \otimes \Lambda^{2} \mathbb{W} \otimes \mathbb{E} \stackrel{\operatorname{Id} \otimes \sigma}{\longrightarrow} \mathbb{W} \otimes \mathbb{E} \stackrel{\epsilon}{\rightarrow} \mathbb{E}
$$

is equal to $-\frac{n-1}{2} \epsilon: \mathbb{W} \otimes \mathbb{E} \rightarrow \mathbb{E}$. 
Proof. Let us perform this computation using abstract indices. For this purpose, if we write $\epsilon: \mathbb{W} \otimes \mathbb{E} \rightarrow \mathbb{E}$ as $w_{a} \otimes e_{\alpha} \mapsto w_{a} \epsilon^{a}{ }^{\beta} e_{\beta}$, then Lemma 3 says that

$$
\epsilon_{\alpha}^{a}{ }^{\beta} \epsilon_{a \beta}^{\gamma}=-n \delta_{\alpha}^{\gamma} \text {. }
$$

Let us write $\sigma: \Lambda^{2} \mathbb{W} \otimes \mathbb{E} \rightarrow \mathbb{E}$ as $X_{a b} \otimes e_{\alpha} \mapsto X_{a b} \sigma^{a b}{ }_{\alpha}{ }^{\beta} e_{\beta}$, where

$$
\sigma_{\alpha}^{a b}{ }_{\alpha}=-\frac{1}{8}\left(\epsilon_{\alpha}^{a}{ }^{\beta} \epsilon^{b}{ }_{\beta}^{\gamma}-\epsilon^{b}{ }^{\beta} \epsilon^{a}{ }_{\beta}^{\gamma}\right) \text {. }
$$

Then (9) becomes

$$
w_{a} e_{\alpha} \mapsto \epsilon_{\alpha}^{a}{ }^{\beta}\left(-2 g_{a[b} w_{c]} \sigma^{b c}{ }_{\beta}^{\gamma} e_{\gamma}\right)=-2 \epsilon_{\alpha}^{a}{ }^{\beta} w_{c} \sigma_{a}{ }^{c} \beta^{\gamma} e_{\gamma}
$$

and so we are required to show that

$$
-2 \epsilon^{a}{ }_{\alpha}{ }^{\beta} \sigma_{a}{ }^{c} \beta^{\gamma}=-\frac{n-1}{2} \epsilon^{c}{ }^{\gamma}{ }^{\gamma} \text { i.e. } \quad 4 \epsilon^{a}{ }_{\alpha}{ }^{\beta} \sigma_{a}{ }^{c} \beta^{\gamma}=(n-1) \epsilon^{c}{ }^{\gamma}{ }^{\gamma} .
$$

Using (10), we compute:

$$
4 \epsilon_{\alpha}^{a}{ }_{\alpha}^{\beta} \sigma_{a}^{c} \beta^{\gamma}=-\frac{1}{2} \epsilon_{\alpha}^{a}{ }^{\beta}\left(\epsilon_{a \beta} \epsilon^{c} \eta^{\gamma}-\epsilon^{c} \beta^{\eta} \epsilon_{a \eta}{ }^{\gamma}\right)=\frac{n}{2} \epsilon_{\alpha}^{c}{ }^{\gamma}+\frac{1}{2} \epsilon^{a}{ }_{\alpha}^{\beta} \epsilon^{c} \beta^{\eta} \epsilon_{a \eta}{ }^{\gamma} .
$$

To continue, let us write (8) with abstract indices:

$$
\epsilon_{\alpha}^{a}{ }_{\alpha}^{\beta} \epsilon_{\beta}^{b} \epsilon_{\eta}^{c}{ }^{\gamma}-\epsilon_{\alpha}^{b}{ }_{\alpha}^{\beta} \epsilon_{\beta}^{c} \epsilon_{\eta}^{a}{ }^{\gamma}-\epsilon_{\alpha}^{a}{ }_{\alpha}^{\beta} \epsilon_{\beta}^{c}{ }^{\eta} \epsilon_{\eta}^{b}{ }^{\gamma}+\epsilon_{\alpha}^{c}{ }^{\beta} \epsilon_{\beta}^{b}{ }^{\eta} \epsilon_{\eta}^{a}{ }^{\gamma}=-4 g^{a b} \epsilon_{\alpha}^{c}{ }^{\gamma}+4 g^{a c} \epsilon_{\alpha}^{b}{ }^{\gamma} \text {. }
$$

Tracing over $a$ and $b$ and using (10) gives

$$
-n \epsilon^{c}{ }_{\alpha}^{\gamma}-\epsilon^{a}{ }_{\alpha}^{\beta} \epsilon^{c}{ }_{\beta}^{\eta} \epsilon_{a \eta}{ }^{\gamma}-\epsilon^{a}{ }_{\alpha}{ }^{\beta} \epsilon^{c}{ }^{\eta}{ }^{\eta} \epsilon_{a \eta}{ }^{\gamma}-n \epsilon_{\alpha}^{a}{ }^{\gamma}=-4 n \epsilon_{\alpha}^{c}{ }^{\gamma}+4 \epsilon_{\alpha}^{c}{ }^{\gamma} .
$$

If follows that

$$
\epsilon_{\alpha}^{a}{ }^{\beta} \epsilon^{c}{ }^{\eta} \epsilon_{a \eta}^{\gamma}=(n-2) \epsilon_{\alpha}^{c} \gamma
$$

and hence that

$$
4 \epsilon_{\alpha}^{a}{ }^{\beta} \sigma_{a}^{c}{ }^{\gamma}{ }^{\gamma}=\frac{n}{2} \epsilon_{\alpha}^{c}{ }^{\gamma}+\frac{n-2}{2} \epsilon_{\alpha}^{c}{ }^{\gamma}=(n-1) \epsilon_{\alpha}^{c}{ }^{\gamma},
$$

as required.

Theorem 2. Let $\mathbb{E}$ denote that representation of $\operatorname{Spin}(n)$ corresponding to (6). Let $E$ denote the corresponding bundle on a conformal spin manifold induced from the orthonormal frames of $\Lambda^{1}[1]$. Then there is a conformally invariant first order linear differential operator

$$
E\left[-\frac{n-1}{2}\right] \stackrel{D}{\rightarrow} E\left[-\frac{n+1}{2}\right]
$$

whose symbol is induced by $\epsilon: \mathbb{W} \otimes \mathbb{E} \rightarrow \mathbb{E}$.

Proof. Proposition 1 ensures that the statement of this theorem makes sense and now Proposition 2 ensures that the criterion of Theorem 1 is satisfied.

Remark 1. This operator $D$ extends the Dirac operator from Clifford analysis on $\mathbb{R}^{n}$ to a general spin manifold. Elements of its kernel are referred to as 'monogenic functions'.

Remark 2. Almost no representation theory is needed here - we have only used that $\operatorname{Spin}(n)$ is the simply-connected connected Lie group whose Lie algebra is $\mathfrak{s o}(n)$. In fact, we shall see in the next section that spin is essential - the representation (6) of $\mathfrak{s o}(n)$ does not arise from an action of $\mathrm{SO}(n)$. The proof of Theorem 2 is purely computational and the only ingredients in this computation are (8) and (10) both of which follow easily from Lemma 1. In fact, the particular constant in Lemma 3 (leading to (10)) is unimportant. We would obtain the same result (with the same conformal weight) from $g_{a b} \otimes e \mapsto \kappa e$ for any non-zero $\kappa$. 


\section{Clifford algebras and the classical Dirac operator}

The Dirac operator from Clifford analysis is usually introduced via the Clifford algebra $\mathcal{C} \ell(\mathbb{W})$, defined as the tensor algebra $\bigotimes^{\bullet} \mathbb{W}$ modulo the two-sided ideal generated by

$$
v \otimes w+w \otimes v+2\langle v, w\rangle \quad \forall v, w \in \mathbb{W} .
$$

Writing the multiplication in $\mathcal{C} \ell(\mathbb{W})$ as juxtaposition, there is a canonical identification

$$
\mathbb{E} \equiv \Lambda^{\bullet} \mathbb{W} \stackrel{\simeq}{\longrightarrow} \ell(\mathbb{W}) \quad \text { by } \quad v \wedge w \mapsto \frac{1}{2}(v w-w v) .
$$

The conclusion (7) of Lemma 1 is precisely that $\epsilon: \mathbb{W} \otimes \mathbb{E} \rightarrow \mathbb{E}$ extends to a representation $\mathcal{C} \ell(\mathbb{W})$, namely to a homomorphism of algebras $\mathcal{C} \ell(\mathbb{W}) \rightarrow \operatorname{End}(\mathbb{E})$. Equivalently, if we transport the Clifford algebra structure from $\mathcal{C} \ell(\mathbb{W})$ to $\Lambda^{\bullet} \mathbb{W}$ using (11) then (5) gives the Clifford product between $\mathbb{W}$ and $\Lambda \bullet \mathbb{W}$ and so the action of $\mathcal{C} \ell(\mathbb{W})$ on $\mathbb{E}$ induced by $\epsilon$ becomes the action of $\mathcal{C} \ell(\mathbb{W})$ on itself by left multiplication. For further details see [13].

A standard rationale for introducing the Clifford algebra is in providing a concrete realisation of $\operatorname{Spin}(n)$, namely as a subgroup of the group of invertible elements in $\mathcal{C} \ell\left(\mathbb{R}^{n}\right)$. Then it is clear that $\mathbb{E}$ under $(11)$ is a real representation of $\operatorname{Spin}(n)$ - simply restrict the action of $\mathcal{C} \ell\left(\mathbb{R}^{n}\right)$ to $\operatorname{Spin}(n)$.

The basic spin representations of $\mathfrak{s o}(n)$ are complex representations. Hence, in order to make contact with $\mathbb{E}$ as an $\mathfrak{s o}(n)$-module given by (6), it is necessary to complexify. Let us suppose that $n$ is even. Then we may write $\mathbb{C W}$ as the direct sum of two totally null subspaces, using the complexified metric as a dual pairing between them. Specifically, let us take

$$
\left.\mathbb{C W}=\mathbb{U} \oplus \mathbb{U}^{*} \ni \alpha+\beta \quad \text { so that } \quad\|\alpha+\beta\|^{2}=2 \alpha\right\lrcorner \beta .
$$

Let use abstract indices to write elements of $\mathbb{U}$ as $\alpha_{a}$ and elements of $\mathbb{U}^{*}$ as $\beta^{a}$, without being alarmed that the index $a$ now runs only over half the range that it did in previous sections. Forms now decompose according to 'type'. Specifically, $\mathbb{C E}=\Lambda \bullet \mathbb{C W}$ and

$$
\Lambda^{r} \mathbb{C} \mathbb{W}=\bigoplus_{p+q=r} \Lambda^{p} \mathbb{U} \otimes \Lambda^{q} \mathbb{U}^{*}
$$

Splitting the formulae (1) according to this decomposition, we find

$$
\begin{aligned}
& \text { for } \Lambda^{p} \mathbb{U} \otimes \Lambda^{q} \mathbb{U}^{*} \ni \omega \rightsquigarrow \underbrace{\omega_{b c \cdots d}}_{p} \overbrace{f g \cdots h}^{q}, \\
& \left\{\begin{array}{l}
\left.(\alpha+\beta) \wedge \omega \leadsto \alpha_{[a} \omega_{b c \cdots d]}{ }^{f g \cdots h}+(-1)^{p} \beta^{[e} \omega_{b c \cdots d} f g \cdots h\right], \\
(\alpha+\beta)\lrcorner \omega \rightsquigarrow p \beta^{b} \omega_{b c \cdots d}^{f g \cdots h}+(-1)^{p} q \alpha_{f} \omega_{b c \cdots d} f g \cdots h .
\end{array}\right.
\end{aligned}
$$

Consequently, as a complexification of the action of $\mathbb{W}$ on $\mathbb{E}=\Lambda \bullet \mathbb{W}$ given by (5) we obtain an action of $\mathbb{C} \mathbb{W}$ on $\Lambda \bullet \mathbb{U} \otimes \Lambda^{\bullet} \mathbb{U}^{*}$ given by

$$
(\alpha+\beta) \cdot \omega \rightsquigarrow \alpha_{[a} \omega_{b c \cdots d]}{ }^{f g \cdots h}+(-1)^{p} \beta^{[e} \omega_{b c \cdots d}{ }^{f g \cdots h]}-p \beta^{b} \omega_{b c \cdots d} f g \cdots h-(-1)^{p} q \alpha_{f} \omega_{b c \cdots d}{ }^{f g \cdots h} .
$$

In accordance with (7) and (12), or as may be verified by direct computation, this action has the property that

$$
(\alpha+\beta) \cdot(\alpha+\beta) \cdot \omega=-2 \alpha_{a} \beta^{a} \omega .
$$

There is, however, another action with this property. Specifically, it is easily verified that

$$
(\alpha+\beta): \omega \leadsto \sqrt{2}\left(\alpha_{[a} \omega_{b c \cdots d]}^{f g \cdots h}-p \beta^{b} \omega_{b c \cdots d} f g \cdots h\right) \Longrightarrow(\alpha+\beta):(\alpha+\beta): \omega=-2 \alpha_{a} \beta^{a} \omega .
$$


Proposition 3. There is an automorphism of $\Lambda^{\bullet} \mathbb{U} \otimes \Lambda^{\bullet} \mathbb{U}^{*}$ that converts $(\alpha+\beta) . \omega$ into $(\alpha+\beta): \omega$ for all $\alpha+\beta \in \mathbb{C W}$.

Proof. More precisely, we want to find $\Phi: \Lambda^{\bullet} \mathbb{U} \otimes \Lambda^{\bullet} \mathbb{U}^{*} \rightarrow \Lambda^{\bullet} \mathbb{U} \otimes \Lambda^{\bullet} \mathbb{U}^{*}$, an invertible linear transformation, so that

$$
(\alpha+\beta): \Phi(\omega)=\Phi((\alpha+\beta) . \omega) \quad \forall(\alpha+\beta) \in \mathbb{U} \oplus \mathbb{U}^{*}=\mathbb{C} \mathbb{W} \quad \text { and } \quad \omega \in \Lambda^{\bullet} \mathbb{U} \otimes \Lambda^{\bullet} \mathbb{U}^{*}
$$

Let us take

$$
\Phi(1) \equiv 1+\delta_{b}^{f}+\delta_{[b}^{[f} \delta_{c]}^{g]}+\cdots+\delta_{\left[b^{[f}\right.}^{[f} \delta_{c}^{g} \cdots \delta_{d]}^{h]}+\cdots .
$$

Then, in order for (13) to hold, we should have $\alpha: \Phi(1)=\Phi(\alpha .1)=\Phi(\alpha)$ whence

$$
\Phi\left(\alpha_{b}\right)=\sqrt{2}\left(\alpha_{b}+\alpha_{[b} \delta_{c]}^{f}+\alpha_{[b} \delta_{c}^{f} \delta_{d]}^{g}+\cdots\right)
$$

Similarly, we should have $\beta: \Phi(1)=\Phi(\beta .1)=\Phi(\beta)$, which forces

$$
\Phi\left(\beta^{f}\right)=-\sqrt{2}\left(\beta^{f}+2 \beta^{[f} \delta_{b}^{g]}+3 \beta^{[f} \delta_{b}^{g} \delta_{c}^{h]}+\cdots\right) .
$$

Now, however, there is something to check because $\alpha . \beta+\beta . \alpha=-2 \alpha_{a} \beta^{a}$ and so for consistency it must be that

$$
\alpha: \Phi(\beta)+\beta: \Phi(\alpha)=-2 \alpha_{a} \beta^{a} \Phi(1) .
$$

This is readily verified as follows

$$
\begin{aligned}
& \alpha_{b}: \Phi\left(\beta^{f}\right)=-2\left(\alpha_{b} \beta^{f}+2 \alpha_{[b} \beta^{[f} \delta_{c]}^{g]}+\cdots\right) \\
& \beta^{f}: \Phi\left(\alpha_{b}\right)=-2\left(\beta^{a} \alpha_{a}+2 \beta^{a} \alpha_{[a} \delta_{b]}{ }^{f}+3 \beta^{a} \alpha_{[a} \delta_{b}{ }^{f} \delta_{c]}{ }^{g}+\cdots\right) \\
&=-2\left(\beta^{a} \alpha_{a}+\beta^{a} \alpha_{a} \delta_{b}^{f}-\beta^{a} \alpha_{b} \delta_{a}^{f}+\beta^{a} \alpha_{a} \delta_{[b}^{f} \delta_{c]}^{g}-2 \alpha_{[b} \beta^{[f} \delta_{c]}^{g]}+\cdots\right) \\
& \Longrightarrow \alpha_{b}: \Phi\left(\beta^{f}\right)+\beta^{f}: \Phi\left(\alpha_{b}\right)=-2 \beta^{a} \alpha_{a}\left(1+\delta_{b}^{f}+\delta_{[b}^{f} \delta_{c]}^{g}+\cdots\right)=-2 \alpha_{a} \beta^{a} \Phi(1) .
\end{aligned}
$$

So far, we know $\Phi$ on $\mathbb{C} \oplus \mathbb{C W}$. But now we may use the desired property

$$
\Phi(v . w)=v: \Phi(w) \quad \text { for } \quad v, w \in \mathbb{C} \mathbb{W}
$$

of $\Phi$ to extend its definition to $\mathbb{C} \oplus \mathbb{C W} \oplus \Lambda^{2} \mathbb{C W}$ : it is only necessary for consistency to check (14) and, similarly, that

$$
\alpha: \Phi(\alpha)=0 \quad \text { and } \quad \beta: \Phi(\beta)=0 .
$$

Next one uses the desired property (13) to extend the definition of $\Phi$ to $\bigoplus_{r=0}^{3} \Lambda^{r} \mathbb{C W}$ and so on by induction. The details are left to the reader.

Remark 3. For those readers who know the theory of Clifford algebras, we have in $\Lambda^{\bullet} \mathbb{C W}$ and $\Lambda^{\bullet} \mathbb{U} \otimes \Lambda^{\bullet} \mathbb{U}^{*}=\operatorname{End}(\mathbb{U})$ two different realisations of the Clifford algebra $\mathcal{C} \ell(\mathbb{C W})$. The mapping $\Phi$ constructed in the proof of Proposition 3 is the unique unital isomorphism between them.

The geometric consequences of Proposition 3 are as follows. Recall that the Dirac operator $D$ in the sense of Clifford analysis was built in $\S 3$ from (5) and (6) both of which are defined in 
terms of $v$.e for $v \in \mathbb{W}$ and $e \in \mathbb{E} \equiv \Lambda^{\bullet} \mathbb{W}$. We have just seen that, in case $n$ is even, we can write

$$
\mathbb{C E}=\Lambda^{\bullet} \mathbb{C} \mathbb{W}=\Lambda^{\bullet}\left(\mathbb{U} \oplus \mathbb{U}^{*}\right)=\Lambda^{\bullet} \mathbb{U} \otimes \Lambda^{\bullet} \mathbb{U}^{*}
$$

Therefore, Proposition 3 may be viewed as providing an automorphism $\Phi$ of $\mathbb{C E}$ so that

$$
\Phi(v \cdot e)=v: \Phi(e) \quad \forall v \in \mathbb{C} \mathbb{W} \text { and } e \in \mathbb{C E} \text {. }
$$

In particular, this is true for $v \in \mathbb{W} \hookrightarrow \mathbb{C W}$. Though it is essential to complexify $\mathbb{W}$ and also to choose a splitting $\mathbb{C W}=\mathbb{U} \oplus \mathbb{U}^{*}$ in order to define $\Phi$ as a complex linear automorphism of $\mathbb{C E}$ and though it is also necessary in order to write down the formula

$$
(\alpha+\beta): \omega \leadsto \sqrt{2}\left(\alpha_{[a} \omega_{b c \cdots d]}^{f g \cdots h}-p \beta^{b} \omega_{b c \cdots d}{ }^{f g \cdots h}\right) \quad \text { for } \quad\left\{\begin{array}{l}
\alpha+\beta \in \mathbb{U} \oplus \mathbb{U}^{*}=\mathbb{C W}, \\
\omega \in \Lambda \bullet \mathbb{U} \otimes \Lambda^{\bullet} \mathbb{U}^{*}=\mathbb{C},
\end{array}\right.
$$

it is not necessary for $v$ to be complex in order that $v: \omega$ be perfectly well-defined. The key point to observe about (15) is that, when viewed on $\Lambda^{\bullet} \mathbb{U} \otimes \Lambda^{\bullet} \mathbb{U}^{*}$, the action $\omega \mapsto v: \omega$ is entirely on $\Lambda^{\bullet} \mathbb{U}$ with $\Lambda^{\bullet} \mathbb{U}^{*}$ as a passenger. It follows that the complexification of the representation (6) of $\mathfrak{s o}(n)$ is isomorphic to $\mathbb{S} \otimes \Lambda^{\bullet} \mathbb{U}^{*}$ where $\mathbb{S}$ is $\Lambda^{\bullet} \mathbb{U}$ regarded as an $\mathfrak{s o}(n)$-module according to

$$
\mathfrak{s o}(n) \otimes \mathbb{S}=\Lambda^{2} \mathbb{W} \otimes \Lambda \bullet \mathbb{U} \ni v \wedge w \otimes \omega \mapsto-\frac{1}{8}(v: w: \omega-w: v: \omega) \in \mathbb{S} .
$$

This action manifestly preserves the splitting $\Lambda^{\bullet} \mathbb{U}=\Lambda^{\text {even }} \mathbb{U} \oplus \Lambda^{\text {odd }} \mathbb{U}$. It is the standard spin representation

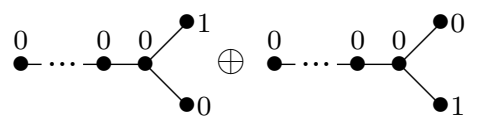

of $\mathfrak{s o}(n)$. In any case, the geometric import of these observations is that there is a complex vector bundle $S$ defined on an arbitrary spin manifold such that the differential operator $D: E \rightarrow E$ defined in $\S 3$, when acting on complex-valued sections, becomes

$$
\mathbb{C} \otimes_{\mathbb{R}} E \cong S \otimes \mathbb{C}^{N} \stackrel{\not D \otimes \mathrm{Id}}{\longrightarrow} S \otimes \mathbb{C}^{N} \cong \mathbb{C} \otimes_{\mathbb{R}} E .
$$

Here $\mathbb{C}^{N}$ denotes the trivial bundle of rank $N=2^{n / 2}$ obtained as the induced bundle from the trivial representation of $\operatorname{Spin}(n)$ on $\mathbb{U}^{*}$. In other words, the Dirac operator $D$ from Clifford analysis may be viewed simply as $2^{n / 2}$-copies of an operator $\not D: S \rightarrow S$. The operator $\not D$ is the classical Dirac operator. There is, however, an awkward proviso to this conclusion, namely that $D$ and $\not D$ should be acting on complex-valued sections. The theory of real spin-bundles is quite complicated [13]. These particular complications are simply avoided in Clifford analysis by using the real operator $D$ instead.

Similar conclusions hold in the odd-dimensional case:

$$
\mathbb{C E}=\Lambda^{\bullet} \mathbb{C W} \cong \bullet \ldots \stackrel{0}{0} \cdot \ldots \mathbb{C}^{N} \Longrightarrow \mathbb{C} \otimes_{\mathbb{R}} E \cong S \otimes \mathbb{C}^{N} \quad \text { and } \quad D=\not D \otimes \mathrm{Id},
$$

where $\not D$ is the classical Dirac operator in odd dimensions and $N=2^{(n+1) / 2}$.

In fact, all complications are avoided by using the operator $D$. This 'one-size-fits-all' approach avoids complex bundles and, at the same time, there is no need to treat even-dimensional and odd-dimensional manifolds differently. The integral formulae for monogenic functions developed in [3], for example, are obtained in a uniform dimension-free manner.

The main point of this article is to provide a simple formulation and naïve computational proof of Theorem 2 and its main consequence, the conformal invariance of monogenic functions. But the conformal invariance of the classical Dirac operator is another consequence. In arbitrary dimensions, this invariance was first shown by Kosmann-Schwarzbach [14]. 


\section{Rarita-Schwinger operators and their generalisations}

Having defined and established the conformal invariance of the Dirac operator in the context of Clifford analysis, it is reasonably straightforward to do the same thing for the classical RaritaSchwinger operator [5] and its symmetric analogues [6]. Throughout this section $\mathbb{E}$ is to be regarded as an $\mathfrak{s o}(n)$-module under $(6)$.

For the Rarita-Schwinger operator, define a representation $\mathbb{F}$ of $\mathfrak{s o}(n)$ via the exact sequence

$$
0 \rightarrow \mathbb{F} \rightarrow \mathbb{W} \otimes \mathbb{E} \stackrel{\epsilon}{\rightarrow} \mathbb{E} \rightarrow 0
$$

bearing in mind that $\epsilon$ is a homomorphism of $\mathfrak{s o}(n)$-modules in accordance with Proposition 1. Lemma 3 provides a canonical splitting of this sequence. Specifically, using abstract indices as in the proof of Proposition 2, we define $\Pi: \mathbb{W} \otimes \mathbb{E} \rightarrow \mathbb{F}$ by

$$
T_{a \alpha} \stackrel{\Pi}{\longmapsto} T_{a \alpha}+\frac{1}{n} \epsilon_{a \alpha}{ }^{\beta} \epsilon^{c}{ }_{\beta}^{\gamma} T_{c \gamma} .
$$

Finally, to define the Rarita-Schwinger operator via Theorem 1, we consider the $\mathfrak{s o}(n)$-module homomorphism $\theta: \mathbb{W} \otimes \mathbb{F} \rightarrow \mathbb{F}$ given as the composition

$$
\mathbb{W} \otimes \mathbb{F} \hookrightarrow \mathbb{W} \otimes \mathbb{W} \otimes \mathbb{E} \stackrel{\tilde{\epsilon}}{\rightarrow} \mathbb{W} \otimes \mathbb{E} \stackrel{\Pi}{\rightarrow} \mathbb{F}
$$

where $\tilde{\epsilon}(w \otimes v \otimes e)=v \otimes w \cdot e$ or, using abstract indices,

$$
T_{a b \alpha} \stackrel{\tilde{\epsilon}}{\longmapsto} \epsilon_{\alpha}^{b}{ }^{\beta} T_{b a \beta} .
$$

Let us write $\tau$ for the representation of $\mathfrak{s o}(n)=\Lambda^{2} \mathbb{W}$ on $\mathbb{F}$. By construction, it is the restriction to $\mathbb{F}$ of the action

$$
\Lambda^{2} \mathbb{W} \otimes \mathbb{W} \otimes \mathbb{E} \ni X \otimes w \otimes e \mapsto X w \otimes e+w \otimes \sigma(X \otimes e) \in \mathbb{W} \otimes \mathbb{E}
$$

on $\mathbb{W} \otimes \mathbb{E}$.

Proposition 4. The composition

$$
\mathbb{W} \otimes \mathbb{F} \stackrel{\iota \otimes \mathrm{Id}}{\longrightarrow} \mathbb{W} \otimes \Lambda^{2} \mathbb{W} \otimes \mathbb{F} \stackrel{\operatorname{Id} \otimes \tau}{\longrightarrow} \mathbb{W} \otimes \mathbb{F} \stackrel{\theta}{\rightarrow} \mathbb{F}
$$

is equal to $-\frac{n-1}{2} \theta: \mathbb{W} \otimes \mathbb{F} \rightarrow \mathbb{F}$.

Proof. In full, we may expand this composition as

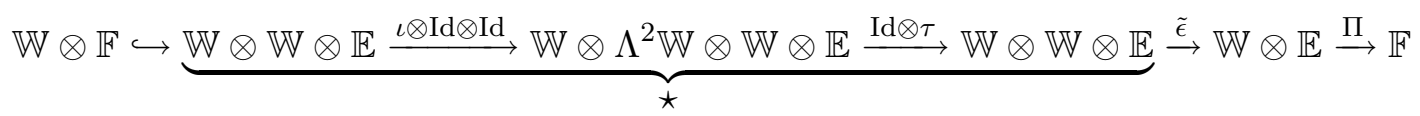

and, according to (17), the homomorphism $\star$ is the sum of two parts, namely

$$
\mathbb{W} \otimes \mathbb{W} \otimes \mathbb{E} \stackrel{\iota \otimes I d \otimes I d}{\longrightarrow} \mathbb{W} \otimes \Lambda^{2} \mathbb{W} \otimes \mathbb{W} \otimes \mathbb{E} \longrightarrow \mathbb{W} \otimes \mathbb{W} \otimes \mathbb{E}
$$

and

$$
\mathbb{W} \otimes \mathbb{W} \otimes \mathbb{E} \stackrel{\iota \otimes I d \otimes I d}{\longrightarrow} \mathbb{W} \otimes \Lambda^{2} \mathbb{W} \otimes \mathbb{W} \otimes \mathbb{E} \longrightarrow \mathbb{W} \otimes \mathbb{W} \otimes \mathbb{E}
$$

in which the boxed vector spaces are passengers. The first one is

$$
T_{a b \alpha} \mapsto-2 g_{a[b} T_{c] d \alpha} \mapsto T_{b a \alpha}-g_{a b} T_{c \alpha}^{c}
$$


and composing with $\tilde{\epsilon}$ gives

$$
T_{a b \alpha} \mapsto \epsilon_{\alpha}^{b}{ }^{\beta}\left(T_{a b \beta}-g_{a b} T_{c \beta}^{c}\right)=-\epsilon_{a \alpha}{ }^{\beta} T_{d \beta}^{d},
$$

when acting on $\mathbb{W} \otimes \mathbb{F}$. Evidently, this is in the kernel of $\Pi$ and so (18) makes no contribution to the overall composition. On the other hand (19) can be continued to

$$
\mathbb{W} \otimes \mathbb{W} \otimes \mathbb{E} \stackrel{\iota \otimes \mathrm{Id} \otimes \mathrm{Id}}{\longrightarrow} \mathbb{W} \otimes \Lambda^{2} \mathbb{W} \otimes \mathbb{W} \otimes \mathbb{E} \longrightarrow \mathbb{W} \otimes \mathbb{W} \otimes \mathbb{E} \stackrel{\tilde{\epsilon}}{\longrightarrow} \mathbb{W} \otimes \mathbb{E}
$$

which has already been computed in Proposition 2. It is $-\frac{n-1}{2} \tilde{\epsilon}$. Therefore, composing with $\Pi$ gives $-\frac{n-1}{2} \theta$, as advertised.

Theorem 3. Let $F$ denote the bundle defined on a conformal spin manifold corresponding to the representation $\mathbb{F}$ of $\mathfrak{s o}(n)$. Then there is a conformally invariant first order linear differential operator

$$
F\left[-\frac{n-1}{2}\right] \stackrel{D}{\rightarrow} F\left[-\frac{n+1}{2}\right]
$$

whose symbol is induced by $\theta: \mathbb{W} \otimes \mathbb{F} \rightarrow \mathbb{F}$.

Proof. Proposition 4 ensures that the criterion of Theorem 1 is satisfied.

The operators in this theorem are the Rarita-Schwinger operators in the context of Clifford analysis. The symmetric analogues of [6] are obtained by a similar construction starting with the exact sequence

$$
0 \rightarrow \mathbb{F}_{j} \rightarrow \bigodot^{j} \mathbb{W} \otimes \mathbb{E} \stackrel{\operatorname{Id} \otimes \epsilon}{\longrightarrow} \bigodot^{j-1} \mathbb{W} \otimes \mathbb{E} \rightarrow 0,
$$

generalising (16). Details are left to the reader.

\section{$6 \quad$ Flat structures and comparison of notations}

The operators that we have constructed in previous sections are conformally invariant in the 'curved' setting. Explicitly, this means that for each operator there is a universal formula in terms of a chosen Riemannian metric and its Levi-Civita connection so that, for an arbitrary metric, using the same formula with any metric in the same conformal class gives the same operator. This is a very strong notion of invariance. There are several weaker notions one of which is to restrict attention to conformally flat metrics, asking only for a local formula in terms of a flat metric from the conformal class and only that the result be invariant under arbitrary flat-to-flat conformal rescalings. Evidently, an invariant operator in the fully curved sense gives rise to an invariant operator in the conformally flat sense as just defined. That curved invariance is strictly stronger, however, was demonstrated by Graham [12] who showed that the operator

$$
\Delta^{3}: \Lambda^{0}[1] \rightarrow \Lambda^{0}[-5] \quad \text { on } \quad \mathbb{R}^{4},
$$

where $\Delta$ is the Laplacian for the standard flat metric on $\mathbb{R}^{4}$ does not arise from a curved invariant operator although it is easily verified to be invariant under flat-to-flat conformal rescalings (see also [11]).

In any case, the Dirac operator is conformally invariant in the curved case and therefore invariant under flat-to-flat conformal rescalings. Such rescalings are scarce. In fact, the only way that they can arise is by so-called 'Möbius transformations', i.e. the transformations obtained from the action of $\mathrm{SO}(n+1,1)$ on the $n$-sphere and viewed in $\mathbb{R}^{n}$ by stereographic projection. Hence, monogenic functions locally defined on $\mathbb{R}^{n}$ are invariant under Möbius transformations. 
This is a well-known phenomenon from Clifford analysis usually attributed to the invariance of the generalised Cauchy integral formula [3] on $\mathbb{R}^{n}$ under Möbius transformations. Monogenic functions on conformally flat spin manifolds such as cylinders and tori provide a natural extension [15, 16] of automorphic forms from the realm of complex analysis. The $n$-sphere is a spin manifold homogeneous under its conformal motions, finitely covered by the group $G=\mathrm{SO}(n+1,1)$ (as indicated below and fully explained, for example, in [9]). It follows that the Dirac operator is $G$-invariant on $S^{n}$ (some authors write $G$-equivariant). This is yet another sense of 'conformal invariance' and a notion that is purely algebraic - the question of classifying the $G$-invariant operators on $S^{n}$ acting between irreducible homogeneous vector bundles is a (solved) question in representation theory. It is this sense of conformal invariance that is in effect throughout the following discussion.

Finally, for completeness, we present the conformal invariance of the Dirac operator on the flat model in terms of the Dynkin diagram notation of [1]. The conformal sphere $S^{n}$ is a homogeneous space for the Lie group $\mathrm{SO}(n+1,1)$. For convenience we shall take this to mean the connected component of the group of $(n+2) \times(n+2)$ matrices preserving the quadratic form

$$
\left[\begin{array}{ccc}
0 & 0 & 1 \\
0 & \mathrm{Id} & 0 \\
1 & 0 & 0
\end{array}\right]
$$

where Id denotes the $n \times n$ identity matrix. This group acts on $S^{n}$ as its conformal motions and, for a suitable choice of basepoint, $S^{n}=\mathrm{SO}(n+1,1) / P$ where $P$ is the subgroup consisting of matrices of the form

$$
\left[\begin{array}{ccc}
\lambda & \cdot & \cdot \\
0 & \mathrm{M} & \cdot \\
0 & 0 & \lambda^{-1}
\end{array}\right], \quad \text { for } \quad\left\{\begin{array}{l}
\lambda>0 \\
M \in \mathrm{SO}(n) .
\end{array}\right.
$$

The irreducible real homogeneous bundles on $S^{n}$ are thus parameterised by the representations

$$
\left[\begin{array}{ccc}
\lambda & \cdot & \cdot \\
0 & \mathrm{M} & \cdot \\
0 & 0 & \lambda^{-1}
\end{array}\right] \mapsto \lambda^{-w} \rho(M)
$$

for $w \in \mathbb{R}$ and $\rho$ an irreducible real representation of $\mathrm{SO}(n)$. It is convenient to specify such a representation of $P$ by recording its highest (or lowest) weight as a linear combination of the fundamental weights for $\mathfrak{s o}(n+1,1)$. With the conventions of [1] we find, for example, that

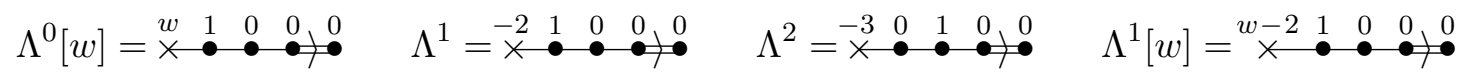

on $S^{9}$ and
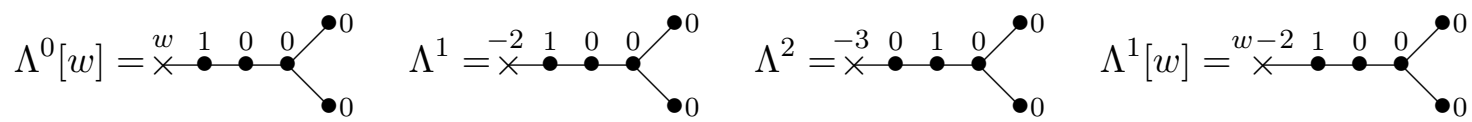

on $S^{10}$. On odd-dimensional spheres $S^{n}$ the Dirac operator acts as

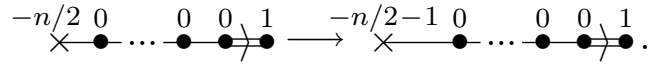

On even-dimensional spheres, however, there are two irreducible Dirac operators:

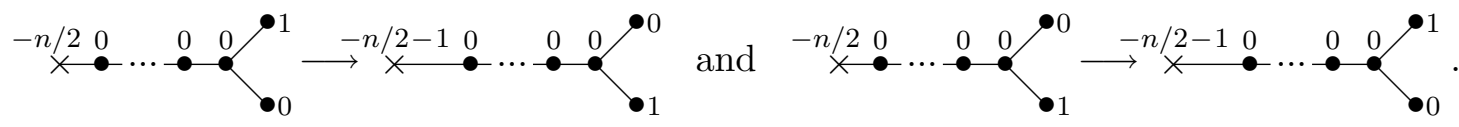

In odd dimensions the generalised Rarita-Schwinger operators of [6] act as

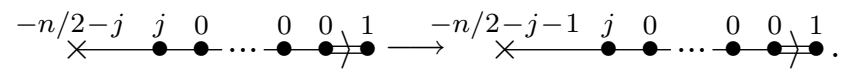




\section{Acknowledgements}

It is a pleasure to acknowledgment useful conversations with Vladimír Souček. He is certainly one person for whom the 'well-known' material in this article is actually known.

Michael Eastwood is a Professorial Fellow of the Australian Research Council. This research was begun during a visit by John Ryan to the University of Adelaide in 2005, which was also supported by the Australian Research Council. This support is gratefully acknowledged. John Ryan also thanks the University of Adelaide for hospitality during his visit.

\section{References}

[1] Baston R.J., Eastwood M.G., The Penrose transform: its interaction with representation theory, Oxford University Press, 1989

[2] Baston R.J., Eastwood M.G., Invariant operators, in Twistors in Mathematics and Physics, London Math. Soc. Lecture Note Ser., Vol. 156, Cambridge University Press, 1990, 129-163.

[3] Brackx F., Delanghe R., Sommen F., Clifford analysis, Research Notes in Math., Vol. 76, Pitman, 1982.

[4] Branson T.P., Differential operators canonically associated to a conformal structure, Math. Scand. 57 (1985), 293-345.

[5] Bureš J., The Rarita-Schwinger operator and spherical monogenic forms, Complex Variables Theory Appl. 43 (2000), 77-108.

[6] Bureš J., Sommen F., Souček V., Van Lancker P., Symmetric analogues of Rarita-Schwinger equations, Ann. Global Anal. Geom. 21 (2002), 215-240.

[7] Calderbank D.M.J., Dirac operators and Clifford analysis on manifolds with boundary, Preprint no. 53, Department of Mathematics and Computer Science, University of Southern Denmark, 1997, available at http://bib.mathematics.dk/preprint.php?lang=en\&id=IMADA-PP-1997-53.

[8] Čap A., Slovák J., Souček V., Invariant operators on manifolds with almost Hermitian symmetric structures, III. Standard operators, Diff. Geom. Appl. 12 (2000), 51-84, math.DG/9812023.

[9] Eastwood M.G., Graham C.R., Invariants of conformal densities, Duke Math. J. 63 (1991), 633-671.

[10] Fegan H.D., Conformally invariant first order differential operators, Quart. J. Math. Oxford (2) 27 (1976), $371-378$.

[11] Gover A.R., Hirachi K, Conformally invariant powers of the Laplacian - a complete nonexistence theorem, J. Amer. Math. Soc. 17 (2004), 389-405, math.DG/0304082.

[12] Graham C.R., Conformally invariant powers of the Laplacian II: nonexistence, J. London Math. Soc. (2) 46 (1992), 566-576.

[13] Harvey F.R., Spinors and calibrations, Academic Press, 1990.

[14] Kosmann-Schwarzbach Y., Propriétés des dérivations de l'algèbre des tenseurs-spineurs, C. R. Acad. Sci. Paris Sér. A-B 264 (1967), A355-A358.

[15] Kraußhar R.S., Ryan J., Clifford and harmonic analysis on cylinders and tori, Rev. Mat. Iberoamericana 21 (2005), 87-110.

[16] Kraußhar R.S., Ryan J., Some conformally flat spin manifolds, Dirac operators and automorphic forms, J. Math. Anal. Appl. 325 (2007), 359-376, math.AP/0212086.

[17] Penrose R., Rindler W., Spinors and space-time, Vol. 1, Cambridge University Press, 1984.

[18] Woodhouse N.M.J., Geometric quantization, Oxford University Press, 1980. 